\title{
Transport of an interacting Bose gas in 1D disordered lattices
}

C. D'Errico, S. Chaudhuri, L. Gori, A. Kumar, E. Lucioni, L. Tanzi, M. Inguscio, and G. Modugno

Citation: AIP Conference Proceedings 1610, 24 (2014); doi: 10.1063/1.4893506

View online: http://dx.doi.org/10.1063/1.4893506

View Table of Contents: http://aip.scitation.org/toc/apc/1610/1

Published by the American Institute of Physics 


\title{
Transport of an interacting Bose gas in 1D disordered lattices
}

\author{
C. D’Errico, S. Chaudhuri, L. Gori, A. Kumar, E. Lucioni, L. Tanzi, M. Inguscio \\ and G. Modugno \\ LENS and Dipartimento di Fisica e Astronomia, Università di Firenze, and CNR-INO \\ 50019 Sesto Fiorentino, Italy
}

\begin{abstract}
We use ultracold atoms in a quasiperiodic lattice to study two outstanding problems in the physics of disordered systems: a) the anomalous diffusion of a wavepacket in the presence of disorder, interactions and noise; b) the transport of a disordered superfluid. a) Our results show that the subdiffusion, observed when interaction alone is present, can be modelled with a nonlinear diffusion equation and the peculiar shape of the expanding density profiles can be connected to the microscopic nonlinear diffusion coefficients. Also when noise alone is present we can describe the observed normal diffusion dynamics by existing microscopic models. In the unexplored regime in which noise and interaction are combined, instead, we observe an anomalous diffusion, that we model with a generalized diffusion equation, where noise- and interaction-induced contributions add each other. b) We find that an instability appearing at relatively large momenta can be employed to locate the fluid-insulator crossover driven by disorder. By investigating the momentum-dependent transport, we observe a sharp crossover from a weakly dissipative regime to a strongly unstable one at a disorder-dependent critical momentum. The set of critical disorder and interaction strengths for which such critical momentum vanishes, can be identified with the separation between a fluid regime and an insulating one and can be related to the predicted zero-temperature superfluid-Bose glass transition.
\end{abstract}

Keywords: Disorder, interaction, transport, diffusion, noise, out-of-equilibrium

PACS: 03.75.Lm, 05.60.-k, 05.45.-a

\section{INTRODUCTION}

Disorder is present everywhere in nature and it can dramatically affect the properties of quantum systems. The transport in superfluids and superconductor, in fact, is strongly affected by the presence of disorder or isolated defects. The most celebrated example was predicted by Anderson [1] for the conductivity in crystals, strongly influenced or eventually suppressed by the presence of even a weak disorder. Moreover the interplay of disorder, non-linearities and noise gives rise to interesting phenomena in a variety of physical systems, including metals and superconductors [2], glasses [3], photonic crystals $[4,5]$ and biological complexes $[6,7,8]$. In the last few decades, in fact, a great interest in the understanding of their complex properties grew up. In particular, interactions, at least in the regime of a weak repulsion, are known to be able to break the Anderson localization induced by the disorder, restoring the coherence and the mobility in an otherwise incoherent, insulating system. This is however a challenging problem, because in most systems it is very difficult to independently control the various contributions. Ultra-cold quantum gases are very promising devices to investigate disordered systems, due to the possibility they offer to precisely tune all the important parameters, including disorder, interactions, noise, thermal and quantum fluctuations $[9,10,11,12]$.

In this contribution to the Transport in Interacting Disordered Systems (TIDS 15) Conference, we outline our recent experimental studies on the transport of an atomic Bose-Einstein condensate in a quasiperiodic optical lattice, to investigate the interplay of the localizing disorder and a weak repulsive interaction across the superfluid-Bose glass transition [13, 14]. In particular, we have investigated how a system that is initially localized by a static disorder is affected by a repulsive interaction or by a temporal noise. A study of the long time expansion dynamics allows us to observe an anomalous diffusion that can be justified in the frame of current theories. We have also studied the short time dynamics of a disordered interacting system confined to one spatial dimension. In this case we observe a non trivial behavior, with dissipation and instabilities, which are determined by the interplay of disorder, interactions and the characteristic phase fluctuations of 1D systems. 


\section{THE DISORDERED INTERACTING SYSTEM}

In the experiment we employ a weakly-interacting Bose-Einstein Condensate in the fundamental band of a quasiperiodic potential, which is realized by perturbing a strong primary optical lattice with a weak, incommensurate, secondary one. Non-interacting particles in the first band of such a potential can be described by the well-known Aubry-André tight-binding Hamiltonian [15]:

$$
H=-J \sum_{j}\left(b_{j}^{\dagger} b_{j+1}+b_{j+1}^{\dagger} b_{j}\right)+\Delta \sum_{j} \cos (2 \pi \delta j) n_{j}
$$

where $b_{j}^{\dagger}, b_{j}$ and $n_{j}=b_{j}^{\dagger} b_{j}$ are the standard on-site creation, destruction and number bosonic operators, $J$ is the kinetic (hopping) energy, $\Delta$ is the quasi-disorder energy and $\delta$ is the ratio of the two lattice spacings. Note that for $\Delta=0$, i.e. in the absence of the secondary lattice, the Aubry-André Hamiltonian is equivalent to the single-particle tight-binding Hamiltonian for a single lattice. The perturbation due to the secondary lattice, in fact, does not change substantially the position of the minima but just shifts the energies in a range $\sim 2 \Delta[16,17]$. This model is known to show Anderson localization for $\Delta>2 J$, with an essentially energy-independent localization length $\xi \sim d / \ln (\Delta / 2 J)$, where $d$ is the main lattice spacing $[16,18]$.

In the experiment we can realize this single-particle regime, and also add a controllable repulsive interaction between the particles, by employing potassium-39 atoms with a magnetically-tunable Feshbach resonance [19, 20]. In the presence of interaction, one needs to introduce an additional term in the Hamiltonian

$$
H_{\text {int }}=U \sum_{j} n_{j}\left(n_{j}-1\right),
$$

where $U$ parameterizes the two-particles interaction energy and $E_{\text {int }} \sim U n(x, t) d$ represents the local interaction per particle, where $n(x, t)$ is the time-dependent density distribution. Such interaction can couple distinct single-particle localized states, allowing for macroscopic transport.

A temporal noise can be introduced by an amplitude modulation of the secondary lattice, with controllable strength $A$ [21]. In this case we have to introduce in the Hamiltonian the following term

$$
H_{\text {noise }}=A \sin \left(\omega_{m} t+\phi_{m}\right) \Delta \sum_{j} \cos (2 \pi \delta j) n_{j}
$$

where the frequency $\omega_{m}$ is randomly varied in a proper interval and the phase $\phi_{m}$ is adjusted to preserve the continuity of the modulation and the sign of its first derivative. This corresponds to a broadband spectrum with a controllable width of the same order of the energy bandwidth of the quasiperiodic potential $(W \approx 2 \Delta+4 J)$, which is able to drive incoherent hopping between the localized states and to allow for macroscopic transport even in a non-interacting system.

We performed two kinds of experiments (Fig. 1). In the first series of experiments we investigated the expansion dynamics of an initially confined wavepacket along a disordered lattice, in the absence of any additional confinement (Fig. 1left). This kind of out-of-equilibrium dynamics has been extensively studied in the theory and we performed its first experimental realization. We were interested in studying the different kinds of dynamics in the presence of disorder (which gives Anderson localization) and a temporal noise (which drives a normal diffusion) and/or a repulsive interaction (able to drive a subdiffusion). In this case, since the system was essentially 3D because of the weak radial confinement $(\sim 50 \mathrm{~Hz})$, the maximum interaction $E_{\text {int }}$ we could reach was of the order of $J$. In this kind of experiments the system was subjected to a long-time dynamics, that we could investigate up to $10 \mathrm{~s}$.

In a second series of experiment we investigated the dynamics of a system initially prepared at the equilibrium in the center of an external trap and then subjected to a sudden shift of the potential (Fig. 1right). We were interested in studying the critical velocity of the superfluid and in understanding how it is affected by the disorder. We investigated this phenomenon in a 1D environment, where both quantum and thermal fluctuations are strongly enhanced, in analogy with quantum wires, but with the additional ability to control the disorder. In this case the system is realized with an additional 2D optical lattice in the radial direction, which splits the 3D Bose-Einstein Condensate in a few hundreds of $1 \mathrm{D}$ quasi-condensates. The strong radial confinement $(50 \mathrm{kHz})$ allows us to reach higher values of the interaction energy $\left(E_{\text {int }} \sim 10 \mathrm{~J}\right)$, but the tight axial confinement $(150 \mathrm{~Hz})$, introduced by the 2D lattice, prevents the observation of the diffusive dynamics we performed in $3 \mathrm{D}$. 


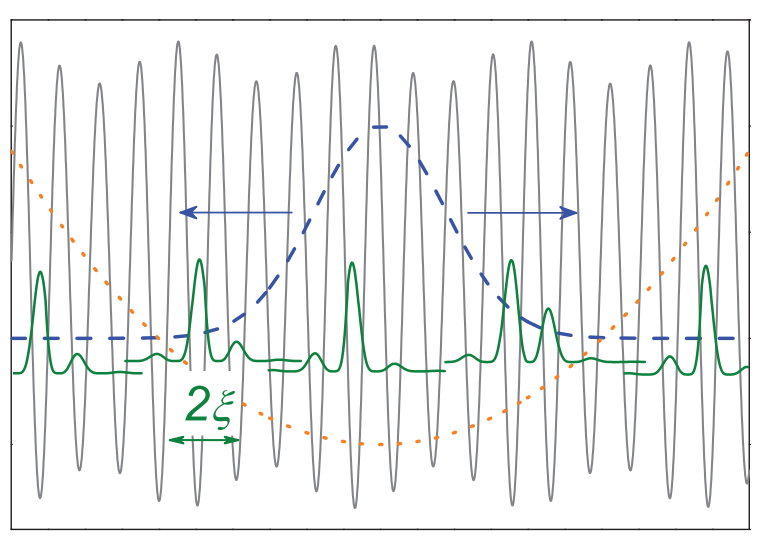

Position

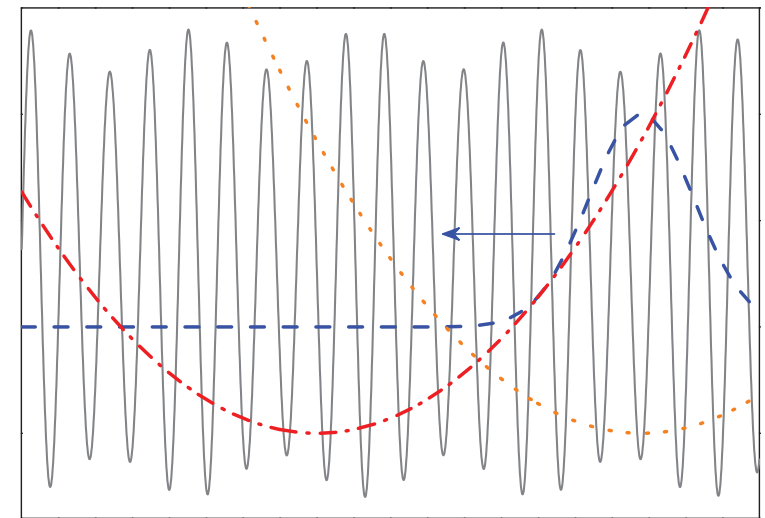

Position

FIGURE 1. Schematic representation of the disordered, interacting system. (left) An initial non-interacting wavepacket (blue dashed line) can be decomposed into exponentially-localized single-particle states (green thick lines) of the quasi-periodic lattice (grey thin line). After having removed the axial harmonic confinement (orange dotted line), a weak interaction or an external noise couple the states and allow an expansion of the wavepacket. Only states separated by one quasiperiod, $d /(\delta-1)$, are shown for clarity. (right) The initial atomic cloud is prepared at equilibrium (blue dashed line) and the initial harmonic confinement (orange dotted line) is suddenly displaced (red dash-dotted line). The atoms start to accelerate in the new trap.

\section{ANOMALOUS DIFFUSION WITH DISORDER, NOISE AND INTERACTION}

To study the dynamics, we initially prepare the condensate close to the ground state of the combined potential of the quasi-periodic lattice with $\Delta>2 J$ and a tight axial harmonic trap, characterized by a Gaussian density distribution $n(x)$. We then suddenly switch off the trap and study the expansion dynamics along the lattice for a variable time, in the presence of an additional radial confinement. In the absence of atom-atom interaction or external noise, the sample is localized in a finite region of the space, as an effect of the Anderson localization mechanism, and we observe only an extremely slow expansion, presumably due to technical noise (Fig. 2). A weak interaction introduces a coherent coupling of single-particle localized states and inhibits localization by giving rise to a subdiffusion, essentially due to the density-dependent local diffusion coefficient. A temporal noise breaks the coherence necessary to have the Anderson localization and hence destroys it. Since this noise is density-independent, it is able to induce a diffusive expansion. A typical time evolution of the width of the system for $\Delta>2 J$ in the presence of noise and interaction is shown in Fig. 2.

The disorder-induced localization is broken by the interaction or the noise alone or also by their combination. In all cases, we observe a short-time transient that evolves into an asymptotic behavior, which is different in the three cases. To model the expansion we fit the evolution of the measured square root of the second moment of $n(x, t)$ with the solution of a generalized diffusion equation:

$$
\sigma(t)=\sqrt{\left\langle x^{2}\right\rangle}=\sigma_{0}\left(1+t / t_{0}\right)^{\alpha}
$$

which is expected to correctly model the overall behavior from short time to the asymptotic regime. Here $\sigma_{0}$ is the initial width, $t_{0}$ is a free parameter that represents the crossover time from the short-time dynamics to the asymptotic one, and $\alpha$ is the long-time diffusion exponent which is characteristic of the dynamics and is different in the three cases. In the combined case of noise and interaction, at short time the expansion is strongly affected by the interaction while at longer time it is dominated by the noise. The interaction-assisted diffusion, in fact, tends to vanish as the sample expands and we should expect a long-time crossover to a regime where interactions effects are negligible and the system diffusion is only due to noise. 


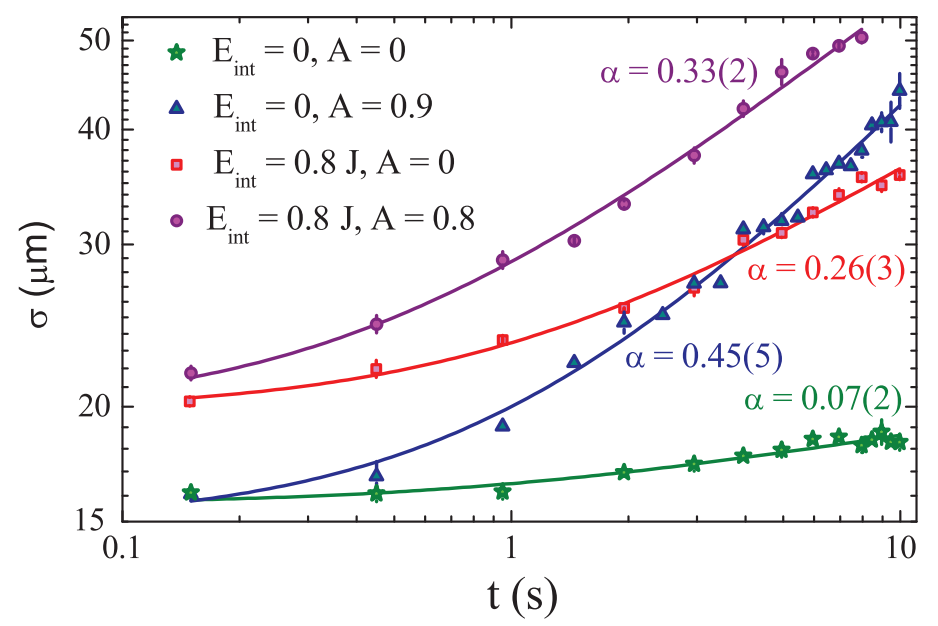

FIGURE 2. Expansion with disorder $(\Delta=4 J)$, noise and interaction. The lines are fits with Eq. (4).

\section{Subdiffusion of an interacting system in a static disordered lattice}

Let's first discuss the effect of a repulsive interaction in a static disordered lattice. As shown in Fig. 2 we essentially observe no expansion if $U=0$, while for a finite $U$ the distribution broadens and changes shape with the increasing time, as already studied both in the theory $[22,23,24,25,26,27,28,29,30]$ and in the experiments [31].

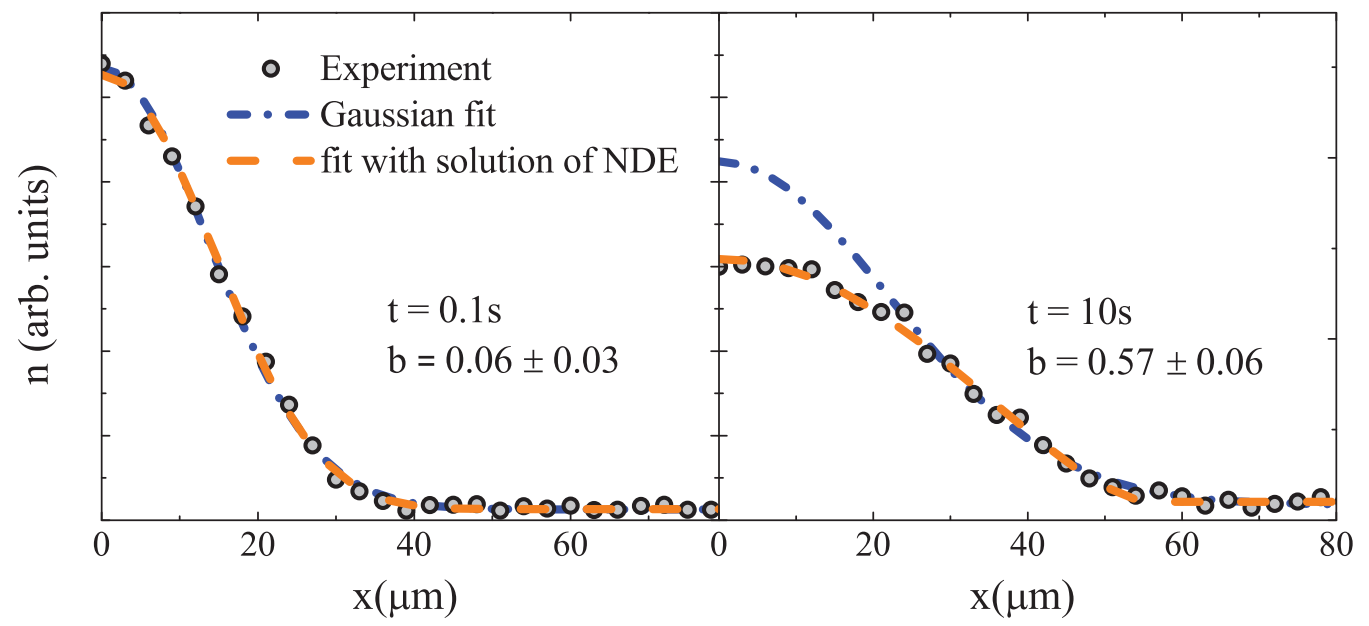

FIGURE 3. Density distribution $n(x, t)$ during the expansion with strong disorder and repulsive interaction. The initial Gaussian distribution (left) evolves into a flat-top distribution at longer times (right). The blue dash-dotted lines are Gaussian fits of the tails of the distribution; the orange dashed lines are fits of the profiles with Eq. (8).

The finite interaction energy breaks the orthogonality between the localized states, weakening the localization. The observed expansion is well fitted with Eq. (4) with a characteristic exponent $\alpha$ in the range 0.2-0.4 [31]. We can describe in a perturbative approach the resulting dynamics as an interaction-assisted coherent hopping between localized states. The diffusion coefficient can be estimated as $D \sim \Gamma \xi^{2}$, where $\xi$ is the natural length scale of the hopping and $\Gamma$ is a coupling rate, that decreases as the system expands. The perturbative approach predicts $D \propto n(x, t)^{\beta} \propto \sigma^{-\beta}$, with $\beta>2$ or $\beta=2$, if respectively $E_{\text {int }} \ll \Delta$ or $E_{\text {int }} \sim \Delta$.

By solving the standard diffusion equation

$$
\frac{\partial n(x, t)}{\partial t}=\frac{1}{2} \frac{\partial}{\partial x}\left(D \frac{\partial n(x, t)}{\partial x}\right)
$$


for a Gaussian distribution $n(x, t) \approx \exp \left[-x^{2} / 2 \sigma(t)^{2}\right]$, we find an evolution of the width $\sigma$ as $d \sigma^{2}(t) / d t=D$, and therefore a time dependence of the form of Eq. (4), with $\alpha=1 / 2$. In this case the dynamics results in a diffusive behavior $(\sigma=\sqrt{D t})$, with a constant diffusion coefficient $D=\sigma_{0}^{2} / t_{0}$.

By still considering a Gaussian, but with a width-dependent diffusion coefficient as $D \propto \sigma^{-\beta}$, Eq. (4) continues to describe the time evolution of the width, but with a time exponent $\alpha=1 /(2+\beta)$. In this case the dynamics results in a subdiffusive behavior, i.e. in a diffusion with a decreasing instantaneous diffusion coefficient

$$
D(t)=2 \alpha \frac{\sigma_{0}^{\alpha^{-1}}}{t_{0}} \sigma(t)^{2-\alpha^{-1}}
$$

While the subdiffusive expansion of the width is well described by this simplified heuristic model of the microscopic dynamics, both in the numerical observations [30] and in the experimental ones [31], a little or no analysis is available for the evolution of the overall shape of $n(x, t)$. We can compare the evolution of the density distribution during the subdiffusive expansion with a solution of a general nonlinear diffusion equation (NDE) of the form:

$$
\frac{\partial n(x, t)}{\partial t}=\frac{\partial}{\partial x}\left(D_{0} n^{a}(x, t) \frac{\partial n(x, t)}{\partial x}\right),
$$

where the diffusion coefficient is explicitly density-dependent. The NDE is usually studied in the asymptotic limit, where its solution is not able to describe the clear change of the shape we observe at short-time expansion. We built the following approximate solution of the NDE:

$$
n(x, t)= \begin{cases}B\left(1-\frac{b(t) x^{2}}{w(t)^{2}}\right)^{1 / b(t)} & |x|<w(t) / \sqrt{b(t)} \\ 0 & |x| \geq w(t) / \sqrt{b(t)}\end{cases}
$$

where $B=B(b, w)$ is an appropriate normalization coefficient and $b(t)=a(1-\exp (-t / \tau))$ is a time-dependent exponent, which provides an accurate interpolation between the initial Gaussian shape and the known asymptotic distribution. As shown in Fig. 3 we found a qualitative good agreement between the experimental evolution of the shape and the generalized solution in Eq. (8), which confirms the hypothesis of a density-dependent diffusion coefficient [32].

\section{Quantum diffusion of a disordered interacting system in the presence of noise}

While the weak interaction destroys localization by inducing a subdiffusive behavior, a temporal noise is known to drive an incoherent hopping between the localized states, which results in a diffusive expansion, as predicted by theory $[33,34,35,36,37]$ and observed in experiments [5, 38, 39, 40, 41].

In the presence of noise alone, in fact, we typically observe an expansion well fitted by Eq. (4) with an exponent $\alpha=0.45$ (5) (Fig. 2), consistent with the normal diffusion, and that the shape $n(x)$ keeps being Gaussian at all times [21]. The measured diffusion coefficient $D=\sigma_{0}^{2} / t_{0}$ shows a clear dependence both on the noise $A$ and disorder $\Delta / J$ strengths. Using a perturbative approach, in the limit of a noise bandwidth equal to the lattice bandwidth, we predict [21]

$$
D=\xi^{2} \Gamma \approx \frac{A^{2} J}{3 \hbar} \frac{(\xi+d)^{2}}{1+e^{d / \xi}} .
$$

which is in good agreement with the experimental observations (Fig. 4).

As shown in Fig. 5, in the presence of both noise and interaction we typically observe a non trivial expansion that is globally faster than for noise or interaction alone, but has an exponent $\alpha$, extracted from the fit with Eq. 4 (purple solid line in Fig. 5), which is intermediate between the two ones measured for interaction alone (red line) and noise alone (blue line). In the combined case, in fact, $\alpha$ varies in the range 0.3-0.5, depending on the relative value of the interaction energy $E_{i n t}$ and the noise amplitude $A$. The observed anomalous diffusion, that is a transient to normal diffusion, can be modelled with a generalized diffusion equation (GDE) for $\sigma^{2}(t)$

$$
\frac{d \sigma^{2}(t)}{d t}=D_{\text {noise }}+D_{\text {int }}(t) .
$$



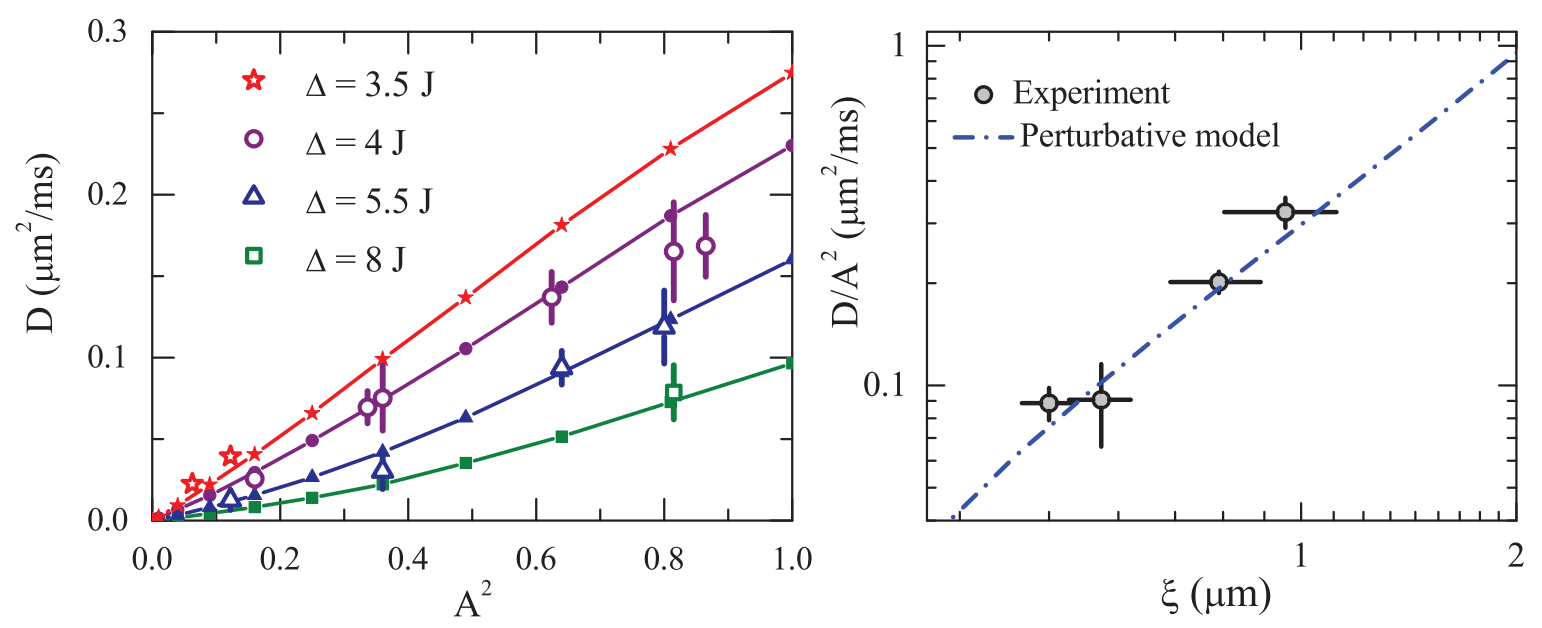

FIGURE 4. Noise-induced normal diffusion: $\xi$ and $A$ dependence of the diffusion coefficient $D$, for $J=150 \mathrm{~Hz}$. Open symbols are the experimental data and filled ones (left) are numerical simulations. Solid lines (left) are a guide to the eyes for numerical simulations. Dash-dotted line (right) is the perturbative prediction from Eq. (9).

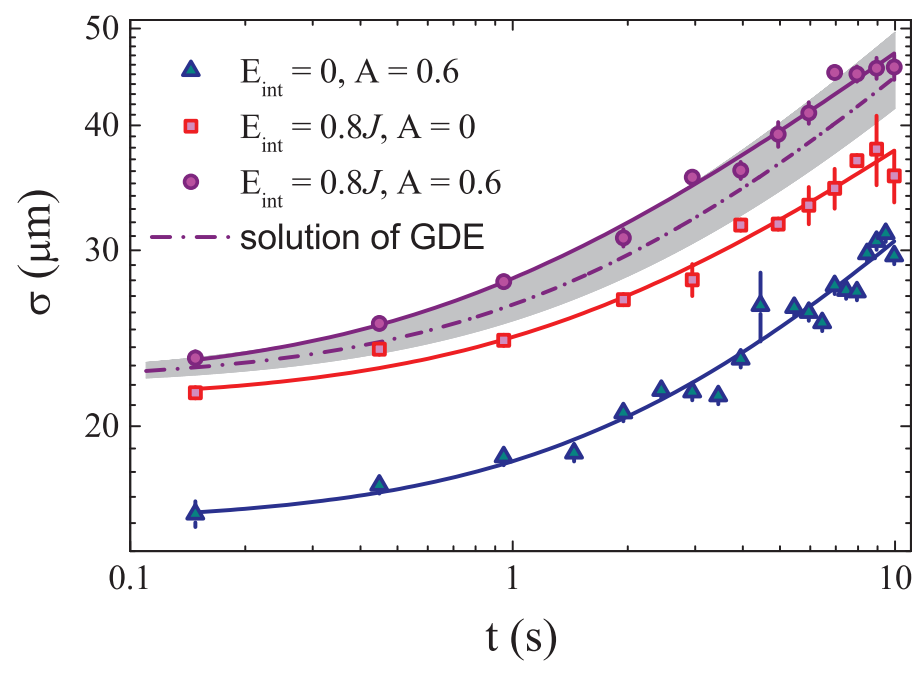

FIGURE 5. Noise and interaction additive anomalous diffusion. Measured time evolution of the width for noise alone (triangles), interaction alone (squares), or both (circles), for $\Delta=4 J$. In the three cases, solid lines are fits with Eq. (4). The dash-dotted line is the numerical solution of Eq. (10) using the extracted diffusion coefficients from the fits for interaction alone (red solid line) and noise alone (blue solid line), with the confidence interval shown as a grey area.

where the instantaneous diffusion coefficient is the sum of the two coefficients of the interaction alone (red line) and the noise alone (blue line): $D_{\text {noise }}$ is the time-independent diffusion coefficient due to noise alone, while $D_{\text {int }}(t)$ is the time-dependent diffusion coefficient for the interaction alone (Eq. (6)). The numerical solution of Eq. (10) is shown in Fig. 5 (purple dash-dotted line). We find that such general diffusion equation, which is similar to a generalized diffusion equation theoretically predicted for Brownian motion of classical interacting particles [42], is valid in a wide range of parameters. 


\section{MOMENTUM-DEPENDENT TRANSPORT AT THE FLUID-INSULATOR TRANSITION}

In a second series of experiments we investigate the transport in a strongly-fluctuating $1 \mathrm{D}$ environment, that we realize with 1D ultracold atomic bosons in quasiperiodic lattices [43].

To realize the system, the 3D Bose-Einstein condensate is subjected to a $2 \mathrm{D}$ lattice that creates a series of parallel 1D tubes, resulting in a few hundreds of $1 \mathrm{D}$ quasi-condensates. The quasi-periodic lattice is loaded along the longitudinal direction. The Bose-Hubbard interaction energy $U$ is varied in the range (0.3-10)J and the mean atom number per site $v$, which scales approximately as $U^{-1 / 3}$, varies in the range of 2-4.

To study the transport, the trap center along the vertical direction is suddenly displaced by a small amount $z_{0}=3.9(2) \mu \mathrm{m}$ by switching off a magnetic-field gradient (Fig. 1). After a variable waiting time in which the atoms evolve in the shifted trap, all potentials are suddenly switched off and the momentum distribution $\rho(p)$ is recorded after a free expansion. In a single lattice, in the absence of any dissipation, the atoms would oscillate with a frequency $\omega^{*}=\omega_{z} \sqrt{m / m^{*}} \simeq 2 \pi \times 90 \mathrm{~Hz}$, were $m^{*}$ is the atomic effective mass in the lattice. In the presence of a disorder, by exciting the motion with variable momentum $p$, we observe a sharp crossover at a disorder-dependent critical momentum from a weakly dissipative regime (at low $p$ ) to a strongly unstable one (at large $p$ ). The vanishing of this critical momentum for the observed instability is then employed to locate the fluid-insulator transition driven by disorder, across the interaction-disorder plane.

\section{Transport in non-disordered lattices}

We started our investigation with non-disordered lattices $(\Delta=0)$, where theoretical models are available. A typical observation of the evolution of $\rho(p)$ is shown in Fig.6, and compared to the solution of the semiclassical equations of motion. At short times, the displacement of the peak momentum, $p_{0}$, can be approximated with a damped oscillation $p_{0}(t)=m^{*} \omega^{* 2} z_{0} / \omega^{\prime} \sin \left(\omega^{\prime} t\right) e^{-\gamma^{*} t}$, where $\omega^{\prime}=\sqrt{\omega^{* 2}-\gamma^{* 2}}$ and $\gamma^{*}=\gamma m / m^{*}$, with a damping rate $\gamma=2 \pi \times(20$ 300) Hz. At longer times, as $p_{0}$ increases towards the center of the Brillouin zone $\left(p=h / 2 \lambda_{1}\right)$, we observe a sudden increase of $\gamma$ and of the width of $\rho(p)$. This causes a stopping of the increase of $p_{0}$, followed by a decay towards zero which can be again fit with a much higher damping rate of the order of $1 \mathrm{kHz}$.
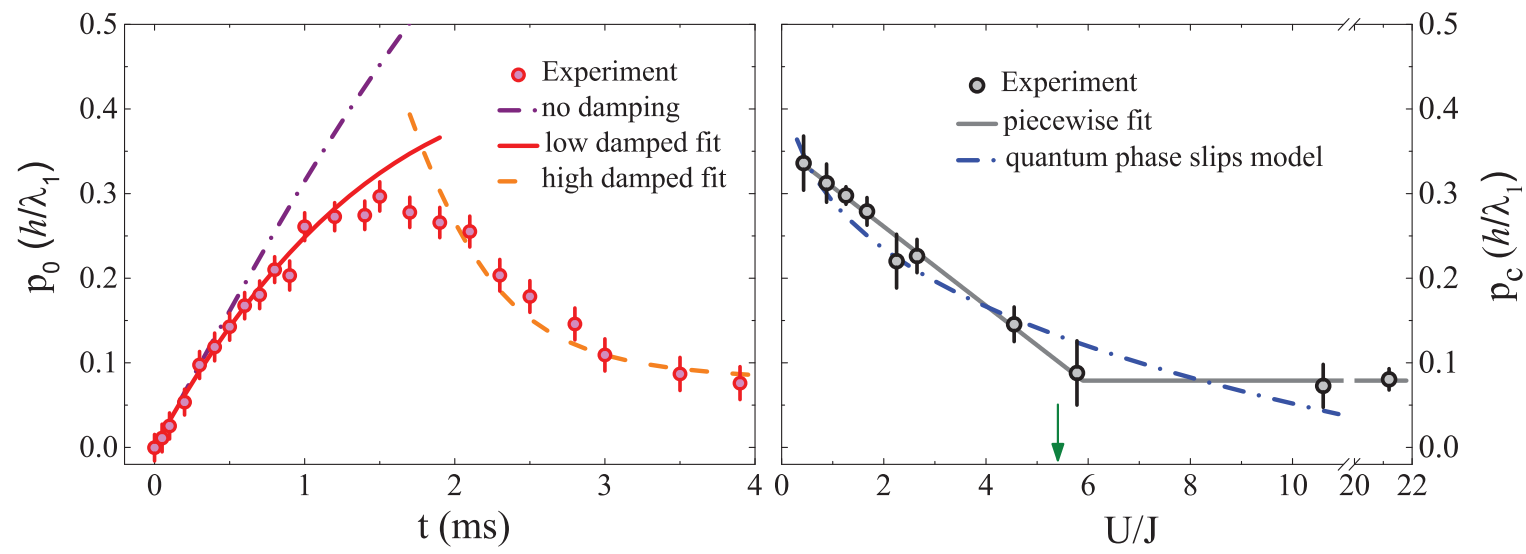

FIGURE 6. Transport in non-disordered lattices. (left) Time evolution of the peak momentum for $U=1.26 J$ and relative observation of the transition from the initial regime of weaker dissipation to the strongly unstable regime at $p_{c}$. (right) Critical momentum $p_{c}$ as a function of the interaction energy. The arrow marks the critical $\mathrm{U} / \mathrm{J}$ for the superfluid-Mott insulator transition (calculated for $v=2[44]$ ).

We estimate a critical momentum $p_{c}$ separating the initial regime of weaker dissipation from the strongly unstable regime, by linearly fitting the difference between the experiment and the fit of the initial oscillation. As shown in Fig. 6, the measured $p_{c}$ features a clear decrease when increasing $U$ at constant $J$. Eventually, $p_{c}$ approaches zero as $U$ approaches the predicted critical value for the Mott insulator. By a piecewise fit of the data, we obtain a critical interaction that is comparable with the value predicted by the theory [44], showing that also in 1D the onset of the 
Mott regime can be detected from a vanishing of $p_{c}$, as in 3D systems [45]. Moreover the decrease of $p_{c}$, accompanied by a corresponding increase of $\gamma$ with $U$, suggests a quantum activation of the phase slip [46, 47, 48].

\section{Transport in disordered lattices}

By introducing a finite $\Delta$ together with the main lattice and by performing the experiment as before we investigated the transport in the presence of disorder. We have in particular studied the weakly-interacting regime, $U / J<3$, where $p_{c}$ for the non-disordered lattice can be very precisely measured. Fig.7 shows how a small $\Delta$ results in a moderate increase of $\gamma$, but also in an anticipated instability. Both changes can be related to the idea that the transport in disorder is dominated by the weakest hopping links, resulting in a smaller effective $J(\Delta)$ that in turn produces an increase of the phase-slip nucleation rates above. For a fixed $U, p_{c}$ features a clear decreasing trend for increasing $\Delta$. Above a critical disorder strength $\Delta_{c}$ of the order of the total interaction energy per atom $v U, p_{c}$ stops decreasing and stays constant at a small value close to that observed in the Mott-insulator regime. This is actually the regime where the disorder can overcome the delocalization effect of the interaction and a weakly-interacting Bose glass is predicted to appear $[49,50]$.
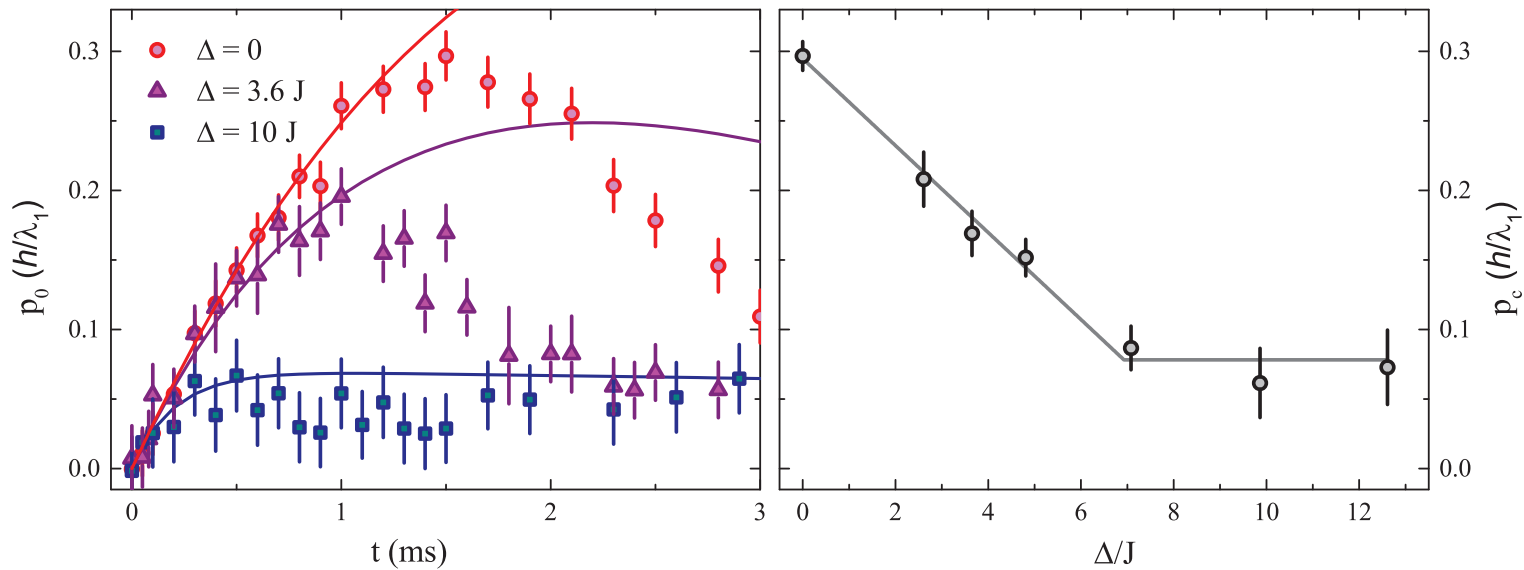

FIGURE 7. Transport in disordered lattices for $U=1.26 \mathrm{~J}$. (left) Time-evolution of the peak momentum for different values of the disorder strength. Lines are fits of the semiclassical motion to the initial oscillation to estimate $p_{c}$. (right) Critical momentum $p_{c}$ as a function of the disorder strength. The line is the piecewise fit used to estimate $\Delta_{c}$.

We studied how $\Delta_{c}$ evolves with $U$. For each $U$, we estimated $\Delta_{c}$ with a piecewise fit of the decreasing $p_{c}(\Delta)$, as shown in Fig.7. The summary of these measurements in Fig.8 shows a clear increase of $\Delta_{c}$ with $U$, indicating that the critical momentum of more strongly interacting systems is less affected by the disorder. The increase of $\Delta_{c}$ is actually fully justified, since the critical disorder strength to enter the Bose glass phase from the superfluid in the regime of weak interactions is expected to scale as $\Delta_{c} / J=A\left(E_{\text {int }} / J\right)^{\alpha}$, where $E_{\text {int }} \simeq v U$ is the total interaction energy per atom, while $A$ and $\alpha$ are coefficients of the order of unity [49, 50,51]. In the absence of an analytical model for the superfluid-Bose glass transition in a quasiperiodic lattice, we fit the experimental data with $\left(\Delta_{c}-2\right) / J=A(v U / J)^{\alpha}$ to account for the critical $\Delta \simeq 2 J$ for localization in the non-interacting system.

The exponent we found, $\alpha=0.86(22)$ is compatible with the mean-field theory prediction $\alpha=1$ for correlated Gaussian disorder in the so called Thomas-Fermi regime, where $E_{i n t}$ is larger than the typical disorder correlation energy $E_{c}$ [50]. For the quasiperiodic lattice we estimate indeed an upper bound $E_{c} \simeq 0.7 J$. The observation is however not incompatible with the prediction $\alpha=\alpha(U)<1$ found in disorder models that include the corrections beyond mean-field [51].

\section{CONCLUSIONS}

Our experiments have provided the first characterization of the transport of a wavepacket in the presence of a controllable disorder, noise and interaction. In the presence of a repulsive interaction we confirmed the subdiffusive 


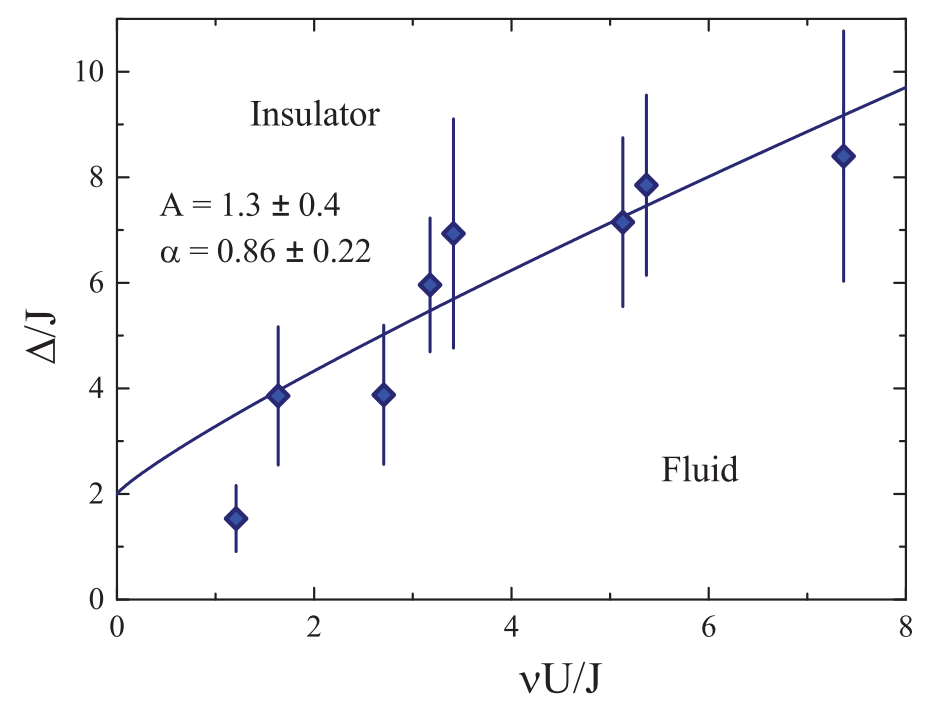

FIGURE 8. Critical disorder to enter the insulating phase as a function of the interaction energy.

nature of the expansion. By connecting the clear evolution of the shape of the expanding density profiles with an approximate solution of the nonlinear diffusion equation, in fact, we confirmed the hypothesis of a microscopic density-dependent diffusion coefficient. In the presence of an external noise we characterized the noise-induced diffusion and its interplay with the interaction induced subdiffusion. We have observed that the complex anomalous diffusion resulting from the simultaneous presence of the two mechanisms can be explained by a simple generalized diffusion equation.

By exploiting the possibility to change the dimensionality of our system, we have also studied the momentumdependent transport of 1D disordered bosons. For non-disordered lattices we observe a rather sharp transition from a weakly dissipative regime at low $p$ to a strongly unstable one at large $p$. When we add a weak disorder we observe an enhanced dissipation and a reduced critical momentum $p_{c}$ for the instability. We observe that for a given interaction strength there is a critical disorder strength above which $p_{c}$ vanishes, which indicates the crossover into an insulating regime. From a set of different measurements we find a crossover line in the interaction-disorder plane that is compatible with the predicted zero-temperature superfluid-Bose glass transition.

The present study was for weak interactions and constant $T$. Future work should explore the role of temperature, easily controllable in our experiment, to investigate the many-body metal-insulator transition [52], and to establish a link with the Luttinger-liquid theory for the superfluid-Bose glass transition for generic $U$ and $\Delta$. Furthermore our system could be used to investigate the barely explored effect of an abrupt quantum quench on a strongly correlated system [53]. We could study, for example, the condition for which a closed system can display local thermalization and the still disputed relation between thermalization and integrability of the system $[54,55,56,57]$. We could also investigate the effect of disorder on this challenging problem.

\section{ACKNOWLEDGMENTS}

We thank B. Deissler for valuable contributions in the experiment and F. Caruso, F. Dalfovo, M. Larcher, M. Moratti, M. Modugno, M.B. Plenio for their theoretical modeling. We acknowledge discussions with B. Altshuler, I. Danshita, S. Fishman, S. Flach, L. Pezzé, G. Shlyapnikov and A. Smerzi. This work was supported by ERC (grants 203479 and 247371) and by MIUR (grants PRIN2009FBKLN and RBFR12NLNA).

\section{REFERENCES}

1. P. W. Anderson, Phys. Rev. 109, 1492 (1958). 
2. Y. Dubi, Y. Meir, and Y. Avishai, Nature 449, 876 (2007).

3. J.-P. Bouchard, and D. S. Dean, Journal de Physique I 5, 265-286 (1995).

4. T. Schwartz, G. Bartal1, S. Fishman and M. Segev, Nature 446, 52 (2007).

5. Y. Lahini, A. Avidan, F. Pozzi, M. Sorel, R. Morandotti, D. N. Christodoulides, Y. Silberberg Phys. Rev. Lett. 100, 013906 (2008).

6. M. Mohseni, P. Rebentrost, S. Lloyd, and A. Aspuru-Guzik, J. Chem. Phys. 129, 174106 (2008)

7. M. B. Plenio and S. F. Huelga, New. J. Phys. 10, 113019 (2008).

8. A. Olaya-Castro, C. F. Lee, F. Fassioli Olsen, F. N. Johnson, Phys. Rev. B 78, 085115 (2008).

9. L. Fallani, C. Fort and M. Inguscio, Advanced in Atomic, Molecular and Optical Physics 56, (2008, New York, Academic).

10. A. Aspect and M. Inguscio, Phys. Today 62, 30 (2009).

11. L. Sanchez-Palencia, M. Lewenstein, Nat. Phys. 6, 87 (2010).

12. G. Modugno, Rep. Progr. Phys. 73, 102401 (2010).

13. T. Giamarchi and H.J. Schulz, Phys. Rev. B 37, 325 (1988)

14. M.P.A.Fisher, P.B. Weichman, G. Grinstein, D.S. Fisher, Phys. Rev. B 40, 546 (1989).

15. S. Aubry, G. André, Ann. Israel Phys. Soc 3, 133 (1980).

16. M. Modugno, New J. Phys. 11, 033023 (2009).

17. V. Guarrera, L. Fallani, J. E. Lye, C. Fort, and M. Inguscio, New J. Phys. 9, 107 (2007).

18. G. Roati, C. D’Errico, L. Fallani, M. Fattori, C. Fort, M. Zaccanti, G. Modugno, M. Modugno, and M. Inguscio, Nature 453, 895 (2008).

19. G. Roati, M. Zaccanti, C. D’Errico, J. Catani, M. Modugno, A. Simoni, M. Inguscio, and G. Modugno, Phys. Rev. Lett. 99, 010403 (2007).

20. C. D'Errico, M. Zaccanti, M. Fattori, G. Roati, M. Inguscio, G. Modugno, and A. Simoni, New J. Phys. 9, 223 (2007).

21. C. D'Errico, M. Moratti, E. Lucioni, L. Tanzi, B. Deissler, M. Inguscio, G. Modugno, M.B. Plenio, and F. Caruso, New J. Phys. 15, 045007 (2013).

22. D. L. Shepelyansky, Phys. Rev. Lett. 70, 1787 (1993).

23. G. Kopidakis, S. Komineas, S. Flach, and S. Aubry, Phys. Rev. Lett. 100, 084103 (2008).

24. A. S. Pikovsky, D. L. Shepelyansky, Phys. Rev. Lett. 100, 094101 (2008).

25. S. Flach, D. O. Krimer, C. Skokos, Phys. Rev. Lett. 102, 024101 (2009).

26. C. Skokos, D. O. Krimer, S. Komineas, and S. Flach, Phys. Rev. E 79, 056211 (2009).

27. H. Veksler, Y. Krivolapov, S. Fishman, Phys. Rev. E 80, 037201 (2009).

28. M. Mulansky, A. Pikovsky, Europhys. Lett. 90, 10015 (2010).

29. M. Larcher, F. Dalfovo, M. Modugno, Phys. Rev. A 80, 053606 (2009).

30. M. Larcher, T. V. Laptyeva, J. D. Bodyfelt, F. Dalfovo, M. Modugno, and S. Flach, New J. Phys. 14, 103036 (2012).

31. E. Lucioni, B. Deissler, L. Tanzi, G. Roati, M. Zaccanti, M. Modugno, M. Larcher, F. Dalfovo, M. Inguscio, G. Modugno, Phys. Rev. Lett. 106, 230403(2011).

32. E. Lucioni, L. Tanzi, C. D’Errico, M. Moratti, M. Inguscio, and G. Modugno, Phys. Rev. E 87, 042922 (2013).

33. A. A. Ovchinnikov and N. S. Erikhman JETP 40, 733 (1975).

34. A. Madhukar and W. Post Phys. Rev. Lett. 39, 1424 (1977).

35. E. Ott, T. M. Antonsen, J. D. Hanson Phys. Rev. Lett. 53, 2187 (1984).

36. S. Fishman and D. L. Shepelyansky Europhys. Lett. 16, 643-648 (1991).

37. D. Cohen Phys. Rev. Lett. 67, 1945 (1991).

38. J. E. Bayfield Chaos 1, 110 (1991).

39. R. Blümel, R. Graham, L. Sirko, U. Smilansky, H. Walther, K. Yamada Phys. Rev. Lett. 62, 341 (1989).

40. M. Arndt, A. Buchleitner, R. N. Mantegna, H. Walther Phys. Rev. Lett. 67, 2435 (1991).

41. D. A. Steck, V. Milner, W. H. Oskay, M. G. Raizen Phys. Rev. E 62, 3461 (2000)

42. G. L. Aranovich and M. D. Donohue, J. Phys. Chem. B 109, 16062 (2005).

43. L. Tanzi, E. Lucioni, S. Chaudhuri, L. Gori, A. Kumar, C. D’Errico, M. Inguscio and G. Modugno, Phys. Rev. Lett 111, 115301 (2013)

44. I. Danshita, A. Polkovnikov, E. Altman, E. Demler, B. Halperin, M.D. Lukin, Phys. Rev. A 84, 063637 (2011).

45. J. Mun, P. Medley, G. K. Campbell, L. G. Marcassa, D. E. Pritchard, and W. Ketterle, Phys. Rev. Lett. 99, 150604 (2007).

46. E. Altman, A. Polkovnikov, E. Demler, B. Halperin, M.D. Lukin, Phys. Rev. Lett. 95, 020402 (2005).

47. A. Polkovnikov, E. Altman, E. Demler, B. Halperin, M.D. Lukin, Phys. Rev. A 71, 063613 (2005).

48. I. Danshita and A. Polkovnikov, Phys. Rev. A 85, 023638 (2012).

49. P. Lugan, D. Clement, P. Bouyer, A. Aspect, M. Lewenstein, and L. Sanchez-Palencia, Phys. Rev. Lett. 98, 170403 (2007).

50. L. Fontanesi, M. Wouters and V. Savona, Phys. Rev. Lett. 103, 030403 (2009).

51. R. Vosk and E. Altman, Phys. Rev. B 85, 024531 (2012).

52. I.L. Aleiner, B.L. Altshuler and G.V. Shlyapnikov, Nat. Phys. 6, 900 (2010).

53. T. Kinoshita, T. Wenger, and D. Weiss, Nature 440, 900 (2006).

54. M. Rigol, V. Dunjko, V. Yurovsky, and M. Olshanii, Phys. Rev. Lett. 98, 050405 (2007).

55. M. Rigol, V. Dunjko, and M. Olshanii, Nature 452, 854 (2008).

56. D. Rossini, A. Silva, G. Mussardo, and G. E. Santoro, Phys. Rev. Lett 102, 127204 (2009).

57. E. Canovi, D. Rossini, R. Fazio, G. E. Santoro, and A. Silva, Phys. Rev. B 83, 094431 (2011). 